\title{
THE MAXIMAL REGULAR IDEAL OF A RING
}

BAILEY BROWN AND NEAL H. MCCOY

1. Introduction. An element $a$ of a ring $R$ is said to be regular if and only if there exists an element $x$ of $R$ such that $a x a=a$. The ring $R$ is regular if and only if each element of $R$ is regular. The concept of a regular ring was introduced by von Neumann $[5,6]^{1}$ who, however, required also that a regular ring have a unit element.

Unless otherwise stated, the word ideal shall mean two-sided ideal, and an ideal in $R$ will be said to be regular if and only if it consists entirely of regular elements of $R$. It is easy to see that a regular ideal $A$ in $R$ is itself a regular ring. For if $a \in A$, there exists an element $x$ of $R$ such that $a x a=a$. It follows that $a x a x a=a$ and $x a x \in A$, so $a$ is regular in the ring $A$.

We shall show that the join of all regular ideals in an arbitrary ring $R$ is a regular ideal, and hence that there exists a unique maximal regular ideal $M=M(R)$ in $R$. The purpose of this note is to establish a few fundamental properties of $M(R)$. Among these are the following "radical-like" properties: (i) $M(R / M(R))=0$, (ii) if $B$ is an ideal in $R$, then $M(B)=B \cap M(R)$, (iii) if $R_{n}$ is the complete matrix ring of order $n$ over $R$, then $M\left(R_{n}\right)=(M(R))_{n}$. A special case of this last result is that $R_{n}$ is regular if and only if $R$ is regular. This was proved by von Neumann [6], but we shall include a very simple proof of this fact.

It is well known that every regular ring has zero (Jacobson) radical $J$. For the rest of the introduction it is assumed that $R$ is a ring such that $R / J$ is regular. We note that this condition is satisfied if, for example, the right ideals of $R$ satisfy the descending chain condition. Thus $M=R$ if and only if $J=0$, and hence, in some sense, $M$ may be considered as an "anti-radical." It is shown in $\$ 4$ that $M=0$ if and only if $R$ is bound to its radical $J$ in the sense of Marshall Hall [2]. Moreover, in $\$ 5$ it is proved that, under the descending chain condition for right ideals, $R$ is expressible as a direct sum

$$
R=M+M^{*},
$$

where $M^{*}$ is the ideal consisting of all elements $a$ of $R$ such that $a M$ $=M a=0$. It follows that $M$ is semi-simple and $M^{*}$ is bound to its radical, and thus this direct sum decomposition coincides with one

Presented to the Society, December 30, 1948; received by the editors January 19, 1949.

1 Numbers in brackets refer to the bibliography at the end of the paper. 
obtained by Marshall Hall [2].

2. Existence and simple properties of $M(R)$. Let $R$ be an arbitrary ring, and $a$ an element of $R$. The following lemma plays a central role in several of our proofs:

LEMMA 1. If $y$ is an element of $R$ such that a-aya is regular, then $a$ is regular.

Proof. If $a-a y a$ is regular, there exists an element $z$ of $R$ such that

$$
(a-a y a) z(a-a y a)=a-a y a .
$$

If we set $x=z-z a y-y a z+y a z a y+y$, a simple calculation shows that $a x a=a$, and thus $a$ is regular, which completes the proof.

We shall indicate by $(a)$ the principal ideal in $R$ generated by $a$. We now prove the following theorem.

THEOREM 1. If $M$ is the set of all elements $a$ of $R$ such that $(a)$ is regular, then $M$ is an ideal in $R$.

Proof. If $z \in M$ and $t \in R$, then $z t \in M$ since $(z t) \subseteq(z)$. Similarly, $t z \in M$. If $z, w \in M$ and $a \in(z-w)$, then $a=u-v$ for some $u$ in (z) and $v$ in $(w)$. Since $(z)$ is regular, $u=u r u$ for some element $r$ of $R$. Then

$$
a-\operatorname{ara}=u-v-(u-v) r(u-v)=-v+u r v+v r u-v r v .
$$

Since $v \in(w)$, this shows that $a-\operatorname{ara} \in(w)$ and is therefore regular. Lemma 1 now implies that $a$ is regular, and hence $z-w \in M$. This completes the proof of the theorem.

It is clear that $M$, being the join of all regular ideals in $R$, and being itself regular, is the unique maximal regular ideal in $R$. It may be remarked that the proof of the above theorem is analogous to the proof of Theorem 1 in Brown and McCoy [1].

We shall next prove the following theorem.

THEOREM 2. If $R$ is any ring, $M(R / M(R))=0$.

Proof. Let $\bar{a}$ denote the residue class modulo $M(R)$ which contains the element $a$ of $R$. If $b \in M(R / M(R))$ and $a \in(b)$, then $\bar{a} \in(b)$. Since $(\bar{b})$ is a regular ideal in $R / M(R), \bar{a}$ is regular. If $\bar{a}=\bar{a} \bar{x} \bar{a}, a$ - axa $\in M(R)$, therefore $a-a x a$ is regular and Lemma 1 implies that $a$ is regular. This shows that every element of $(b)$ is regular, and hence $b \in M(R)$. Thus $b=0$, completing the proof.

Suppose now that $B$ is an ideal in $R$, and let $b$ be an element of $B$ which generates a regular ideal $(b)^{\prime}$ in the $\operatorname{ring} B$. Let $(b)$ be the ideal in $R$ generated by the element $b$, and let 


$$
c=n b+r b+b s+\sum r_{i} b s_{i} \quad\left(n \text { an integer; } r, s, r_{i}, s_{i} \text { in } R\right)
$$

be any element of $(b)$. Since $b$ is regular in $B$ we have $b=b b_{1} b$ for some $b_{1}$ in $B$. Hence

$$
c=n b+\left(r b b_{1}\right) b+b\left(b_{1} b s\right)+\sum\left(r_{i} b b_{1}\right) b\left(b_{1} b s_{i}\right),
$$

and thus $c \in(b)^{\prime}$; therefore $(b)$ is regular since it coincides with $(b)^{\prime}$. This shows that if $b \in M(B)$, then $b \in B \cap M(R)$. Conversely, if $b \in B \cap M(R)$, then $b$ is an element of $B$ which is regular in $R$, and it is easy to see that $b$ is therefore regular in the ring $B$. Since $B \cap M(R)$ is a regular ideal in the ring $B$, it follows that $B \cap M(R)$ $\subseteq M(B)$. We have therefore proved the following theorem.

Theorem 3. If $B$ is an ideal in $R$, then $M(B)=B \cap M(R)$.

3. The maximal regular ideal of a complete matrix ring. In this section we shall prove the following theorem.

THEOREM 4. If $R_{n}$ is the complete matrix ring of order $n$ over $R$, then

$$
M\left(R_{n}\right)=(M(R))_{n} .
$$

First we give an elementary proof of the special case of this result in which $R$ itself is regular, and therefore $R=M(R)$. This result, under the assumption that $R$ has a unit element, is due to von Neumann [6].

Lemma 2. If $R$ is a regular ring, then $R_{n}$ is a regular ring.

The proof of this is in two steps, the first being the proof for $n=2$, and the second the extension to arbitrary $n$. If $r \in R$, let us denote by $r^{\prime}$ an element of $R$ such that $r r^{\prime} r=r$. Now let

$$
A=\left(\begin{array}{ll}
a & b \\
c & d
\end{array}\right)
$$

be an arbitrary element of $\boldsymbol{R}_{\mathbf{2}}$. If we set

$$
X=\left(\begin{array}{ll}
0 & 0 \\
b^{\prime} & 0
\end{array}\right)
$$

and denote $A-A X A$ by $B$, a simple calculation shows that

$$
B=\left(\begin{array}{ll}
g & 0 \\
h & i
\end{array}\right)
$$

for suitable choice of elements $g, h, i$ of $R$. If 


$$
Y=\left(\begin{array}{ll}
g^{\prime} & 0 \\
0 & i^{\prime}
\end{array}\right)
$$

then

$$
C=B-B Y B=\left(\begin{array}{ll}
0 & 0 \\
k & 0
\end{array}\right),
$$

for some element $k$ of $R$. Finally, if

$$
Z=\left(\begin{array}{cc}
0 & k^{\prime} \\
0 & 0
\end{array}\right)
$$

we see that

$$
C-C Z C=0 .
$$

This means that $C$ is regular and hence, by Lemma $1, B$ is regular. Again applying Lemma 1, we see that $A$ is regular, and this completes the proof for $\boldsymbol{n}=2$.

Since $\left(R_{2}\right)_{2} \cong R_{4}$, it follows from the case just proved that $R_{4}$ is regular, and similarly $R_{2^{k}}$ is regular for any positive integer $k$. If now $n$ is an arbitrary positive integer, choose $k$ so that $2^{k} \geqq n$. If $A \in R_{n}$, let $A_{1}$ be the matrix of $R_{2^{k}}$ with $A$ in the upper left-hand corner and zeros elsewhere. Now, as an element of $R_{2^{k}}, A_{1}$ is regular, that is, there exists an element

$$
X=\left(\begin{array}{ll}
B & C \\
D & E
\end{array}\right)
$$

of $R_{2^{k}}$ such that $A_{1} X A_{1}=A_{1}$. However, this implies that $A B A=A$, and hence $A$ is regular. The proof of the lemma is therefore complete.

By the lemma just proved, $(M(R))_{n}$ is a regular ideal in $R_{n}$, and hence $(M(R))_{n} \subseteq M\left(R_{n}\right)$. Conversely, let $A$ be a matrix in $M\left(R_{n}\right)$, and let $a_{i j}$ be a fixed element of $A$. Since $(A)$ is a regular ideal, there exists an element $X$ of $R_{n}$ such that $A=A X A=A X A X A$, and therefore

$$
a_{i j}=\sum_{p, q} t_{p q} a_{p q} s_{p q}
$$

for suitable elements $t_{p q}, s_{p q}$ of $R$. But it is easy to see ${ }^{2}$ that there exists a matrix of $(A)$ with $t_{p q} a_{p q} s_{p q}$ in $(1,1)$ position and zeros elsewhere, and hence an element of $(A)$ with $a_{i j}$ in $(1,1)$ position and zeros elsewhere. Now if $b$ is any element of the principal ideal in $R$

${ }^{2}$ See, for example, Lemma 5 of [1]. 
generated by $a_{i j}$, it is clear that there exists an element $B$ of $(A)$ with $b$ in the $(1,1)$ position and zeros elsewhere. Furthermore, we have $B Y B=B$ for suitable choice of $Y$ in $R_{n}$ since $(A)$ is regular. But this implies that $b y_{11} b=b$, and hence $b$ is regular. This shows that $a_{i j}$ $\in M(R)$, and hence that $M\left(R_{n}\right) \subseteq(M(R))_{n}$, completing the proof of the theorem.

4. Some additional properties of $M(R)$. By the annihilator $B^{*}$ of an ideal $B$ in a ring $R$ is meant the ideal consisting of all elements $a$ of $R$ such that $a B=B a=0$.

THEOREM 5. If $M$ is the maximal regular ideal of a ring $R$ and $J$ is the Jacobson radical of $R$, then $M \cap J=0, J \subseteq M^{*}, M \subseteq J^{*}$, and $M \cap M^{*}=0$. Furthermore, $J$ is the radical of the ring $M^{*}$ and $M$ is the maximal regular ideal of the ring $J^{*}$.

Proof. Since $J$ contains no nonzero idempotent element $[3$, p. 305], $M \cap J=0$. From this it follows that $M J=J M=0$, so $J \subseteq M^{*}$ and $M \subseteq J^{*}$. If $a \in M \cap M^{*}$, then $a=a x a$ for some $x$. But $a \in M$ and $x a$ $\in M^{*}$, hence $a(x a)=0$ and $M \cap M^{*}=0$. The last sentence of the theorem follows from the observation of Perlis [4] that if $B$ is any ideal in $R$, the radical of the ring $B$ is just $B \cap J$, and from the analogous Theorem 3.

Following Marshall Hall [2], we may say that a ring $R$ is bound to its radical $J$ if and only if $J^{*} \subseteq J$.

The next theorem gives, for a class of rings including all those whose right ideals satisfy the descending chain condition, a necessary and sufficient condition that the maximal regular ideal be the zero ideal.

Theorem 6. If $R$ is a ring such that $R / J$ is regular, then $M=0$ if and only if $R$ is bound to $J$.

Proof. If $R$ is bound to $J$, it follows that $M=0$ even without the condition that $R / J$ be regular. For $M \cap J=0$, and this implies, as in Theorem 5, that $M \subseteq J^{*} \subseteq J$. Hence $M=0$.

Conversely, let $R / J$ be regular and $M=0$. We show first by induction that $J \cap J^{* 2}=0$. Suppose that $j \in J$ and that $j=\sum_{i=1}^{n} a_{i} b_{i}$ where $a_{i}, b_{i}$ are in $J^{*}$. It must be proved that $j=0$. In the regular ring $R / J, \bar{a}_{i}$ is regular, so $R$ contains $x_{i}$ such that $a_{i}-a_{i} x_{i} a_{i}=j_{i} \in J$. Since $b_{i} \in J^{*}$, we have

$$
j=\sum_{i=1}^{n}\left(a_{i} x_{i} a_{i}+j_{i}\right) b_{i}=\sum_{i=1}^{n} a_{i} x_{i} a_{i} b_{i} .
$$

If $n=1$, this implies that $j=a_{1} x_{1} a_{1} b_{1}=a_{1} x_{1} j=0$ since $a_{1} \in J^{*}$. If $n \neq 1$, 
then

$$
a_{n} b_{n}=j-\sum_{i=1}^{n-1} a_{i} b_{i}
$$

Thus by (1)

$$
j=\sum_{i=1}^{n-1} a_{i} x_{i} a_{i} b_{i}+a_{n} x_{n}\left(j-\sum_{i=1}^{n-1} a_{i} b_{i}\right)=\sum_{i=1}^{n-1}\left(a_{i} x_{i}-a_{n} x_{n}\right) a_{i} b_{i} .
$$

But the induction hypothesis asserts that if $j=\sum_{i=1}^{n-1} c_{i} d_{i}$ and $c_{i}, d_{i}$ are in $J^{*}$, then $j=0$. Since $\left(a_{i} x_{i}-a_{n} x_{n}\right) a_{i}$ and $b_{i}$ are in $J^{*}$, it follows that $j=0$, and we have proved that $J \cap J^{* 2}=0$. This implies, however, that $J^{* 2}$ is a regular ideal. For if $a \in J^{* 2}$, then in the regular ring $R / J$, the element $\bar{a}$ is regular, that is, for some $x, a-a x a \in J \cap J^{* 2}=0$, so $a$ is regular. Hence $J^{* 2} \subseteq M=0$, from which it follows that $J^{*} \subseteq J$ since the radical contains all nil ideals $[3$, p. 304]. Thus $R$ is bound to $J$ and the proof is complete.

5. A decomposition theorem. In this section we point out the role played by the maximal regular ideal $M$ in a theorem of Hall [2], and incidentally give a new proof of his result.

Lemma 3. If an ideal $B$ in a ring $R$ has a unit element $e$, then

$$
R=B+B^{*} \text {. }
$$

Proof. The existence of a unit element in $B$ implies that $B \cap B^{*}=0$. If $x \in R$, then $e x+x e \in B$ and hence $(e x+x e) e=e(e x+x e)$, from which it follows that $x e=e x$ and $e$ is in the center of $R$. Thus the Peirce decomposition

$$
x=e x+(x-e x)
$$

expresses each element $x$ of $R$ as a sum of elements $e x$ of $B$ and $x-e x$ of $B^{*}$, and the desired result is established.

We remark that a right ideal $I$ in the ring $M$ is a right ideal in $R$. For if $a \in I, r \in R$, then $a r \in M$, hence for some element $y$ of $R$, aryar $=a r$. But ryar $\in M$, so ar $\in I$. Thus $I$ is a right ideal in $R$.

From this remark, it follows that if the descending chain condition for right ideals holds in $R$, it holds also in $M$. In the presence of this chain condition, regularity is equivalent to semi-simplicity. Hence $M$ has a unit element, and the first sentence of the following theorem is implied directly by Lemma 3.

THEOREM 7. If a ring $R$ satisfies the descending chain condition for right ideals, then 


$$
R=M+M^{*} .
$$

The ring $M$ is semi-simple and the ring $M^{*}$ is bound to its radical.

The semi-simplicity of $M$ is implied by the regularity of $M$. Since the maximal regular ideal of $M^{*}$ is zero by Theorem 3 , and the chain condition holds in $M^{*}$, it follows from Theorem 6 that $M^{*}$ is bound to its radical.

Hall has shown that a ring $R$ satisfying the descending chain condition for right ideals can be represented in a unique way as the direct sum of a semi-simple ring and a ring which is bound to its radical. The result just established shows that the semi-simple component is precisely the maximal regular ideal $M$ of $R$, and the bound component is the annihilator of $M$.

\section{BIBLIOGRAPHY}

1. B. Brown and N. H. McCoy, The radical of a ring, Duke Math. J. vol. 15 (1948) pp. 495-499.

2. M. Hall, The position of the radical in an algebra, Trans. Amer. Math. Soc. vol. 48 (1940) pp. 391-404.

3. N. Jacobson, The radical and semi-simplicity for arbitrary rings, Amer. J. Math. vol. 67 (1945) pp. 300-320.

4. S. Perlis, $A$ note on the radical of an ideal, Bull. Amer. Math. Soc. Abstract 539-306.

5. J. von Neumann, On regular rings, Proc. Nat. Acad. Sci. U. S. A. vol. 22 (1936) pp. 707-713.

6. - Continuous geometry, Princeton University Lectures, 1936-1937 (Planographed).

Amherst College AND

SMith College 